\title{
Family Medicine Clerkship Teaching About Contraception: Meeting a Need
}

\author{
Dharam Persaud-Sharma ${ }^{1}$, Joseph Burns ${ }^{2}$, Kyle P. Sellers ${ }^{3}$, Marquita Samuels ${ }^{4}$, Sarah Stumbar ${ }^{5}$ \\ 1. Internal Medicine, Kendall Regional Medical Center, Herbert Wertheim College of Medicine, Florida International \\ University, Miami, USA 2. Pediatrics, Cohen Children's Medical Center, New Hyde Park, USA 3. Department of \\ Translational Medicine, Herbert Wertheim College of Medicine, Florida International University, Miami, USA 4. \\ Department of Humanities, Health, and Society, Herbert Wertheim College of Medicine, Florida International \\ University, Miami, USA 5. Family Medicine, Herbert Wertheim College of Medicine, Florida International University, \\ Miami, USA
}

Corresponding author: Kyle P. Sellers, ksell011@med.fiu.edu

\begin{abstract}
Introduction: Women's healthcare including preventive care, obstetric care, and family planning and counseling are important medical education topics that student physicians frequently encounter during their Family Medicine Clerkship in medical training; however, despite its brief emphasis, many students feel underprepared to counsel patients in a clinical setting. With this in mind, a case-based curriculum focused on women's health was developed for students enrolled in the Family Medicine Clerkship.
\end{abstract}

Methods: During the 2018-2019 academic year, 127 students on the Family Medicine Clerkship at the Florida International University Herbert Wertheim College of Medicine (HWCOM) participated in three two-hour sessions on the topics of adolescent preventive health care, obstetric care, and contraceptive counseling. These sessions incorporated cases, role-play activities, and pre-session readings. An optional, anonymous, paper-based pre-session survey of Likert-type questions was administered prior to the first session and a post-session survey of Likert-type questions was given following the final session. IRB exemption was obtained.

Results: The pre-session survey showed that $41.67 \%$ of students agreed or strongly agreed that they were confident in their ability to perform female contraceptive counseling. After the sessions, $92.68 \%$ of students agreed or strongly agreed that the case addressing contraceptive counseling was of clinical value to their learning needs.

Conclusion: Given that less than half of the students felt confident in their counseling abilities prior to these sessions, this curriculum is potentially addressing an unmet educational need. Students found this session to be relevant, indicating that a case-based discussion curriculum that incorporates both pre-session reading materials and role-play interactions may be a promising educational approach.

Received 06/30/2020

Review began 07/13/2020

Review ended 09/07/2020

Published 09/11/2020

(c) Copyright 2020

Persaud-Sharma et al. This is an open

access article distributed under the terms

of the Creative Commons Attribution

License CC-BY 4.0., which permits

unrestricted use, distribution, and

reproduction in any medium, provided the original author and source are credited.
Categories: Family/General Practice, Medical Education

Keywords: undergraduate medical education, women's health, family planning, contraception

\section{Introduction}

Between 2006 and 2010, nearly 43 million women between the ages of 15 and 44 reported receiving gynecologic care, including family planning services and Pap tests during the preceding year [1]. The role of the family physician in addressing women's healthcare is well-established. For example, approximately onethird of pregnant women report seeking care from family physicians [2]. As two-thirds of women of reproductive age in the United States currently use some type of contraception, contraceptive counseling and management is an important skill for any future physician [3].

An analysis of 21 medical schools across the United States found that students looking to gain competency in contraceptive counseling often must undertake family planning electives [4]. Another series of four focus groups of medical students identified lack of time devoted to contraception in didactic education as a primary perceived barrier to education on this topic [5]. As patient-centered contraception counseling leads to increased use, gaining competency in this skill is important for students [6]. While the Society of Teachers of Family Medicine (STFM) National Clerkship Curriculum does not have specific objectives related to contraceptive counseling, this topic is generally addressed in preventive health visits, which are a focus of any Family Medicine curriculum [7].

Case-based discussion has been shown to be an effective teaching strategy in medical education [8]. A longitudinal case-based discussion curriculum that consisted of three two-hour sessions spanning multiple didactic days was implemented in order to ensure that students in the third year Family Medicine Clerkship at the Herbert Wertheim College of Medicine (HWCOM) at Florida International University in Miami were 
receiving adequate exposure to women's healthcare. Topics in women's healthcare that were included in this curriculum included adolescent preventive health counseling, obstetric care, and contraceptive counseling. On the first day, students were introduced to a simulated patient to address her preventive health needs. The second session guided students through a simulated pregnancy cycle; outcomes from these sessions have been published [9]. The third session introduced students to contraceptive counseling as part of the simulated patients' post-partum visit. Before participating in the session, students were provided with two articles for pre-reading materials $[10,11]$. The purpose of this study was to evaluate the enrolled students' perceived need for and perception of this contraceptive counseling session.

\section{Materials And Methods}

During the 2018-2019 academic year, 127 students on the Family Medicine clerkship at the Florida International University HWCOM participated in three two-hour case-based learning sessions regarding adolescent preventive health care, obstetric care, and contraceptive counseling. Each session involved all 19-23 students completing their Family Medicine clerkship and was facilitated by a Family Medicine-trained faculty member with specific expertise in women's health. The learning objectives for the three sessions are detailed in Table 1 .

By the end of these sessions, students should be able to:

Day 1: Preventive Care

. Describe the HEADSSS exam and the essential elements of an adolescent preventative health visit.

Understand the family physician's role in well-patient care; and be able to discuss age- and sex-appropriate preventative care guideline.

Day 2: Obstetric Care

- Explain the management of first trimester bleeding, including the use of and indications for imaging, lab tests, medications, and surgical procedures.

. Articulate the pathophysiology and appropriate management of nausea/vomiting of pregnancy.

Explain the difference between chronic hypertension in pregnancy and pre-eclampsia, including the diagnostic criteria of each.

- List the diagnostic criteria for gestational diabetes and its first-line treatment options, as well as potential complications that may impact the fetus.

Articulate how obstetric care is in alignment with the principles of Family Medicine

Day 3: Post-Partum Care

Counsel a young female patient regarding contraception options in the immediate post-partum period and list other post-partum preventative health concerns.

\section{TABLE 1: Learning objectives for longitudinal contraception case-based learning sessions}

HEADSSS: Home, Education/Employment, Eating, Activities, Drugs, Sexuality, Suicide/Depression, and Safety

An optional, anonymous, paper-based pre-session survey of Likert-type questions evaluating students' confidence regarding contraceptive counseling was administered prior to the first session, and a postsession survey of Likert-type questions assessing students' perceptions of the session was given following the final session. Data was categorized based on whether or not students had already completed their obstetrics/gynecology (OBGYN) clerkship. IRB exemption was obtained.

\section{Results}

All 127 students in the clerkship participated in the case-based learning sessions; $96.3 \%$ completed the presession survey, and 59.8\% completed the post-session survey. At the time of participation, 61 students had not completed their OBGYN clerkship and 54 had. Among the students who had not already completed their OBGYN clerkship, 36.01\% agreed or strongly agreed that they were confident in their ability to perform male contraceptive counseling, and $41.67 \%$ felt similarly about their ability to perform female contraceptive counseling.

Among the students who had completed their OBGYN clerkship, 50.0\% agreed or strongly agreed that they were confident in their ability to perform male contraceptive counseling, and $74.1 \%$ felt similarly about their ability to perform female contraceptive counseling. Responses to the pre-session questions are found in Table 2 . 


\section{Cureus}

Statement

Number (\%)

I am confident in my ability to perform male contraceptive counseling.

Students who had not already completed the OBGYN clerkship ${ }^{a}$

Strongly agree

Agree

Neutral

Disagree

Strongly Disagree

$0(0.00)$

Students who had already completed OBGYN clerkship ${ }^{\mathrm{b}}$

Strongly Agree

5 (9.62)

Agree

$21(40.4)$

Neutral

$16(30.8)$

Disagree

9 (17.3)

Strongly Disagree

1 (1.92)

I am confident in my ability to perform female contraceptive counseling.

Students who had not already completed their OBGYN clerkship ${ }^{c}$

Strongly agree

Agree

$24(40.0)$

Neutral

23 (38.3)

Disagree

$12(20.0)$

Strongly Disagree

$0(0.00)$

Students who had already completed their OBGYN clerkship ${ }^{d}$

Strongly Agree

Agree

$27(50.0)$

Neutral

$12(22.2)$

Disagree

Strongly Disagree

$0(0.00)$

\section{TABLE 2: Students' responses to pre-session survey, categorized based on whether or not} students had already completed their obstetrics/gynecology (OBGYN) clerkship

$a_{n=61}$, response rate: $100 \%$

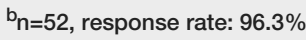

$c_{n}=60$, response rate: $98.4 \%$

$d_{n}=54$, response rate: $100 \%$

93.6\% of students agreed or strongly agreed that the pre-session readings provided them with clinically relevant knowledge, and $96.1 \%$ of students agreed or strongly agreed that the in-person sessions helped them apply the information from the pre-session readings. $92.68 \%$ of the students agreed or strongly agreed that the case was of clinical value to them and their learning needs. Responses to the post-session questions can be found in Table 3 . 


\section{Cureus}

Statement

Number (\%)

The pre-session readings provided me with clinically relevant knowledge $\mathrm{e}^{\mathrm{e}}$

Strongly Agree

Agree

Neutral

Disagree

The Natalie case study helped me to apply the information from the pre-session readings ${ }^{f}$

\section{Strongly Agree}

Agree

Disagree

Natalie's case was of clinical value to me and my learning needs 9

$\begin{array}{lr}\text { Strongly Agree } & 38(46.34) \\ \text { Agree } & 38(46.34) \\ \text { Neutral } & 6(7.32) \\ \text { Disagree } & 0(0.00) \\ \text { Strongly Disagree } & 0(0.00)\end{array}$

\section{TABLE 3: Students' responses to post-session questions}

$e_{n}=78$, response rate: $61.5 \%$

$f_{n}=76$, response rate: $59.8 \%$

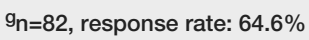

\section{Discussion}

The results of the pre-session survey revealed that many students on the Family Medicine Clerkship were not confident in their ability to perform contraceptive counseling. Although students who had already completed the OBGYN clerkship were more confident than those who had not, approximately half of these students still did not feel confident in their ability to provide male contraceptive counseling. This suggests that there is room to improve the current curriculum, particularly regarding male contraceptive counseling; male contraceptive counseling was not a focus of our curriculum.

Additionally, even after the OBGYN clerkship, approximately one quarter of students reported that they did not feel confident in their ability to provide female contraceptive counseling; this suggests that the OBGYN clerkship alone is not sufficient in providing medical students with this education.

Students had an overwhelmingly positive reaction to this curriculum, with over $90 \%$ of students agreeing that the sessions were of value to their learning needs. This positive reaction may indicate that education regarding contraception is generally insufficient within medical school curricula, especially during clinical years when students are tasked with independently counseling patients. The fact that students found this session to be relevant indicates that a case-based discussion curriculum that incorporates both pre-session reading materials and role-play interactions may be a promising tool in the education of medical students on family planning and counseling.

These results support findings from the literature which indicate that medical students find that the case- 
based learning model enhances their learning $[12,13]$. There is not much literature regarding the effectiveness of case-based learning specifically regarding contraceptive counseling and family planning; however, one study by Weiss et al. demonstrated that case-based learning is useful in education regarding women's health in general [14]. Research has not been conducted on case-based curricula that incorporate role-play interactions and pre-session reading.

Limitations of this study include its cross-sectional design, which precludes an analysis of the long-term impact of this curriculum. Self-selection of the survey may have introduced bias into data to complete the post-session survey. Also, the questions from the pre-session survey were not included in the post-session survey. As such, statistical analysis could not be completed to compare pre-session and post-session confidences.

\section{Conclusions}

This study provides insight into a unique educational approach to contraception counseling and family planning that can be easily implemented in medical school education. The approach was novel in that it included both student-developed pre-session reading materials and role-play simulations during the educational encounter to improve student comfort with counseling patients in a clinical environment. The results of this study indicated that this multi-dimensional approach may be useful in teaching medical students about women's healthcare needs from contraception, hygiene, and throughout pregnancy. Further utilization of this approach will allow students to develop the skills necessary to successfully counsel their patients. Future research should look more directly at student confidence by including related questions in the post-session survey. Further adaptations of this curriculum would be the incorporation of a similar clinical encounter as a structured, end of the year observed clinical skills examination (OSCE).

\section{Additional Information}

\section{Disclosures}

Human subjects: Consent was obtained by all participants in this study. Florida International University Office of Research Integrity issued approval IRB-18-0115-AM01. The Florida International University Office of Research Integrity has approved the following modification(s): $\mathbf{x}$ As Dharam Persaud Sharma and Joseph Burns have recently graduated from Florida International University Herbert Wertheim College of Medicine (FIU HWCOM), Kyle Sellers--a member of the FIU HWCOM class of 2022--will be assisting in reviewing several Family Medicine Clerkship didactic sessions in their place. $\mathbf{~ C I T I ~ B a s i c ~ T r a i n i n g ~ C o m p l e t i o n ~ R e p o r t ~}$ attached for Kyle Sellers. $\mathbf{X}$ Extend the study to the Class of 2021. $\mathbf{~ A s ~ a ~ r e s u l t ~ o f ~ e x t e n d i n g ~ t h e ~ s t u d y ~ t o ~}$ include members of the class of 2021, the number of participants will increase from 135 to 270. This change has been reflected in the informational letter. There are no additional requirements in regards to your study. However, if there are further changes in the protocol after you commence your study, then you are required to resubmit your proposal for review. For further information, you may visit the FIU IRB website at http://research.fiu.edu/irb. Animal subjects: All authors have confirmed that this study did not involve animal subjects or tissue. Conflicts of interest: In compliance with the ICMJE uniform disclosure form, all authors declare the following: Payment/services info: All authors have declared that no financial support was received from any organization for the submitted work. Financial relationships: All authors have declared that they have no financial relationships at present or within the previous three years with any organizations that might have an interest in the submitted work. Other relationships: All authors have declared that there are no other relationships or activities that could appear to have influenced the submitted work.

\section{Acknowledgements}

D.P.S, J.B., and K.S. would like to thank Dr. Gillis, Dr. Rao, and Dr. E. Gray for their contributions to this paper. We would also like to thank the Florida International University Undergraduate Honors Bioethics students for their assistance, contributions, and efforts in making this paper possible.

\section{References}

1. Martinez G, Chandra A, Febo-Vazquez I, Mosher W: Use of family planning and related medical services among women aged 15-44 in the United States: National Survey of Family Growth, 2006-2010. Natl Health Stat Report. 2013, 68:

2. Kozhimannil KB, Fontaine P: Care from family physicians reported by pregnant women in the United States . Ann Fam Med. 2013, 11:350-354. 10.1370/afm.1510

3. Daniels K, Abma JC: Current contraceptive status among women aged 15-49: United States, 2015-2017. NCHS Data Brief. 2018, 327:

4. Veazey K, Nieuwoudt C, Gavito C, Tocce K: Student perceptions of reproductive health education in US medical schools: a qualitative analysis of students taking family planning electives. Med Educ Online. 2015, 20:28973. 10.3402/meo.v20.28973

5. Smith KG, Gilliam ML, Leboeuf M, Neustadt A, Stulberg D: Perceived benefits and barriers to family planning education among third year medical students. Med Educ Online. 2008, 13:4474. 10.3885/meo.2008.Res00250

6. Nobili MP, Piergrossi S, Brusati V, Moja EA: The effect of patient-centered contraceptive counseling in women who undergo a voluntary termination of pregnancy. Patient Educ Couns. 2007, 65:361-368. 


\section{Cureus}

10.1016/i.pec.2006.09.004

7. Society of Teachers of Family Medicine: National Clerkship Curriculum. 2018.

8. McLean SF: Case-based learning and its application in medical and health-care fields: a review of worldwide literature. J Med Educ Curric Dev. 2016, 10.4137/JMECD.S20377

9. Stumbar SE, Minor S, Samuels M: A prenatal standardized patient experience for medical students on their family medicine clerkship. Fam Med. 2018, 50:376-379. 10.22454/FamMed.2018.826159

10. Persaud-Sharma D, Burns J, Trangle J, et al.: An introduction and high yield summary of female contraceptive methods. HWCOM Faculty Publications. 2017, 117:

11. Persaud-Sharma D, Burns J, Trangle J, Gilbert A, Govea M, Lorenzo M, Rao CV: Male contraceptives: a new frontier in contraception medicine. Austin J Reprod Med Infertil. 2017, 4:1048.

12. Thistlethwaite JE, Davies D, Ekeocha S, et al.: The effectiveness of case-based learning in health professional education. A BEME systematic review: BEME Guide No.23. Med Teach. 2012, 34:e421-e444. 10.3109/0142159X.2012.680939

13. George T, Carey RA, Abraham OC, Sebastian T, Faith MF: Trainee doctors in medicine prefer case-based learning compared to didactic teaching. J Family Med Prim Care. 2020, 9:580-4. 10.4103/ifmpc.jfmpc 109319

14. Weiss LB, Levison SP: Tools for integrating women's health into medical education: clinical cases and concept mapping. Acad Med. 2000, 75:1081-1086. 